\title{
A NOTE ON DERIVATIONS OF COMMUTATIVE ALGEBRAS
}

\section{T. ANDERSON}

It is well known that the (solvable) radical of a Lie or Jordan algebra is invariant under all derivations of the algebra if the groundfield is not modular [4] and [5]. In this note we obtain a similar result for commutative power-associative algebras of degree one by following Jacobson's argument in [5] and then appealing to a theorem of Gerstenhaber [3] at that point where the Jordan identity was required.

Our result (Theorem 1) seems useful in classifying simple algebras of degree one which satisfy identities giving rise to derivations of the algebras. For example, an immediate corollary to Theorem 1 is Kleinfeld and Kokoris' determination of simple flexible algebras of degree one [6]. Then in Theorem 2 we characterize the simple degree one algebras which satisfy identities considered by Kosier [7], Osborn [8], and the author [2].

Before giving Theorem 1, it is necessary to state some definitions and elementary identities.

In an algebra $A$ the associator $(x, y, z)$ and commutator $(x, y)$ are defined for each $x, y, z$ in $A$ by the equations $(x, y, z)=(x y) z-x(y z)$ and $(x, y)=x y-y x$. The following identity may easily be verified.

$$
(x y, z)=x(y, z)+(x, z) y+(x, y, z)+(z, x, y)-(x, z, y) .
$$

Given any algebra $A$, we may form a commutative algebra $A^{+}$by letting $A^{+}$be the vector space of $A$ in which a new product $x \circ y$ is defined in terms of the product $x y$ of $A$ by the rule $x \circ y=x y+y x$. By direct calculation, we have

$$
\begin{aligned}
(x \circ y) \circ z-x \circ(y \circ z)= & (x, y, z)+(x, z, y)+(y, x, z)-(y, z, x) \\
& -(z, x, y)-(z, y, x)+(y,(x, z)) .
\end{aligned}
$$

Now we proceed to the theorems.

TheOREM 1. Let $C$ be a commutative power-associative algebra of degree one over an algebraically closed field $F$ of characteristic zero, so that $C=1 F+N$, where $1=$ unity of $C$ and $N$ is the ideal of all the nilpotent elements of $C\left[1\right.$, p. 526]. Then for any derivation $D$ of $C, C^{D} \subseteq N$.

Proof. Jacobson [5] has pointed out that the identity $(w x)^{D}=w^{D} x$

Received by the editors February 26, 1966. 
$+w x^{D}$ can be written in operator form as $R_{x} D=\left(R_{x}, D\right)$, where $R_{u}$ denotes the mapping $a \rightarrow a u$ of $C$, so that trace $R_{x} D=0$ for all $x \in C$.

Moreover, trace $R_{w}=0$ for all $w$ in $N$. For since $C$ is commutative and $w$ is nilpotent, $R_{w}$ is nilpotent [3, Theorem 2], hence trace $R_{w}=0$.

Now suppose that $x$ is an arbitrary element of $C$ and that $x^{D}$ $=\alpha 1+w$, where $\alpha \in F$ and $w \in N$. Then $R_{x} D=\alpha I+R_{w}$ and $0=\operatorname{trace} R_{x} D$ $=\alpha \cdot \operatorname{dim} C+$ trace $R_{w}=\alpha \operatorname{dim} C$. Therefore $\alpha=0$ and $x^{D}=w \in N$.

CoRollary. If $B$ is a simple flexible power-associative algebra of degree one over an algebraically closed field $F$ of characteristic zero, then $B \cong F$ (Kleinfeld-Kokoris [6]).

Proof. Let $C$ be the algebra $B^{+}$. Then $C$ is an algebra of degree one, hence $C=1 F+N$, where $N$ is an ideal of $C$. In other words, $n \circ a \in N$ for all $n \in N, a \in B$. Since $B$ is flexible, the mapping $D: x$ $\rightarrow(x, a)$ is a derivation of $B^{+}$. From Theorem 1 it follows that $C^{D} \subseteq N$, hence, in particular, $(n, a) \in N$. But then, $n a=\frac{1}{2}\{n \circ a+(n, a)\} \in N$, and then $a n=n \circ a-n a \in N$, so that $N$ is an ideal of $B$. Since $N \neq B$ and $B$ is simple, it follows that $N=0$ and $B=1 F \cong F$.

THEOREM 2. Let $A$ be a simple power-associative algebra of degree one over an algebraically closed field $F$ of characteristic zero, and suppose that $A$ satisfies one of the following identities:

$$
\begin{aligned}
& \left(x^{2}, y, z\right)=x \circ(x, y, z), \\
& \left(y, x^{2}, z\right)=x \circ(y, x, z), \\
& \left(z, y, x^{2}\right)=(z, y, x) \circ x .
\end{aligned}
$$

Then $A$ has a basis $\left\{1, u_{1}, \cdots, u_{n}\right\}$ such that

(6) $u_{i} u_{j}=\lambda_{i j} 1$, where $\lambda_{i j}=-\lambda_{j i} \in F, \quad i, j=1, \cdots, n$,

and

$$
\operatorname{det}\left(\lambda_{i j}\right) \neq 0
$$

Moreover,

$$
(x, y, z)=(z, y, x) \text { is an identity in } A .
$$

Conversely, if an algebra $A$ has a basis for which (6) and (7) hold, then $A$ is a simple power-associative algebra satisfying the identities (3), (4), and (5).

REMARKs. Kosier [7] has shown that simple rings with idempotent $\neq 1$ which satisfy (3) and (5) are alternative. Theorem 2 shows that 
in the degree one case, such rings are not, in general, even flexible. For results on algebras satisfying (4), see [2] and [8].

Proof of Theorem 2. We again let $C$ be the algebra $A^{+}$. Then $C$ is an algebra of degree one, and since $C$ and $A$ are the same vector space, $C=A=1 F+N$, where $N$ is the ideal of nilpotent elements of $C$ and $N \circ A \subseteq N$.

If the algebra $A$ satisfies the identity (3), then as a linearization of (3) we have the identity $(w \circ x, y, z)=w \circ(x, y, z)+(w, y, z) \circ x$, so that for all $y, z \in A$, the mapping $D: x \rightarrow(x, y, z)$ is a derivation of $C$. Therefore $(x, y, z) \in N$ for all $x, y, z \in A$, because of Theorem 1 . Similarly, by linearizing (4) and (5) we obtain derivations of $C$, so that in any case we may say that

$$
(A, A, A) \subseteq N \text {. }
$$

Then from the fact that $N \circ A \subseteq N$, it follows from (2) and (9) that $(N,(N, N)) \subseteq N$, and since $N \circ(N, N) \subseteq N$, we have that $N(N, N) \subseteq N$ and $(N, N) N \subseteq N$. Applying now (1) and (9), we see that $\left(N^{2}, N\right) \subseteq N$, hence from $N^{2} \circ N \subseteq N$, we obtain

$$
N^{2} N \subseteq N
$$

Now we can show that

$$
N^{2} \cap N \text { is an ideal of } A \text {. }
$$

Clearly, $N^{2} \cap N$ is a subspace of $A$. Suppose $z \in N^{2} \cap N$ and let $a=\alpha 1+w, \alpha \in F, w \in N$, be an arbitrary element of $A$. Then $z a$ $=\alpha z+z w \in N+N^{2} N \subseteq N$ because of (10), and $z a$ is clearly contained in $N^{2}$. Therefore $z a \in N^{2} \cap N$. Similarly, $a z \in N^{2} \cap N$, which completes the proof of (11).

Since $A$ is simple, we must have $N^{2} \cap N=0$. Now suppose that $\left\{u_{1}, \cdots, u_{n}\right\}$ is a basis for $N$. Then $u_{i} u_{j}=\lambda_{i j} 1+z_{i j}$, where $\lambda_{i j} F$ and $z_{i j} \in N$. Let $w$ be an arbitrary element of $N$. Then $z_{i j} w=\left(u_{i} u_{j}\right) w$ $-\lambda_{i j} w \in N^{2} \cap N$ because of (10). Therefore $z_{i j} w=0$. Similarly, $w z_{i j}=0$. But then $z_{i j} F$ is an ideal of $A$ which is contained in $N$. From the fact that $A$ is simple we conclude that $z_{i j}=0$. Thus $u_{i} u_{j}=\lambda_{i j} 1$, and since $u_{i} \circ u_{j} \in N, \lambda_{i j}=-\lambda_{i j}$. We have proved (6).

Suppose that $\operatorname{det}\left(\lambda_{i j}\right)=0$. Then there exist $\xi_{1}, \cdots, \xi_{n}$ in $F$, not all $=0$, such that

$$
\sum_{j=1}^{n} \lambda_{i j} \xi_{j}=0, \quad i=1, \cdots, n .
$$

Let $x=\sum_{j=1}^{n} \xi_{j} u_{j}$. Then for each $i=1, \cdots, n, u_{i} x=\sum_{j=1}^{n} \xi_{j} u_{i} u_{j}$ 
$=\sum_{j=1}^{n} \lambda_{i j} \xi_{j} \cdot 1=0=-x u_{i}$. Therefore $x F$ would be a proper ideal of $A$, contrary to simplicity. Hence $\operatorname{det}\left(\lambda_{i j}\right) \neq 0$.

Having the multiplication table for the basal elements of $A$, it is quite easy to verify (8), so the proof will be omitted. The converse is straightforward and is left to the reader for verification.

\section{REFERENCES}

1. A. A. Albert, $A$ theory of power-associative commutative algebras, Trans. Amer. Math. Soc. 69 (1950), 503-527.

2. C. T. Anderson, On an identity common to Lie, Jordan, and quasi-associative algebras, Ph.D. dissertation, Ohio State University, Columbus, 1964.

3. M. Gerstenhaber, On nilalgebras and linear varieties of nilpotent matrices. II, Duke Math. J. 27 (1960), 21-31.

4. N. Jacobson, Lie algebras, Interscience, New York, 1962.

5. - Derivation algebras and multiplication algebras of semi-simple Jordan algebras, Ann. of Math. 50 (1949), 866-874.

6. E. Kleinfeld and L. Kokoris, Flexible algebras of degree one, Proc. Amer. Math. Soc. 13 (1962), 891-893.

7. Frank Kosier, $A$ generalization of alternative rings, Trans. Amer. Math. Soc. 112 (1964), 32-42.

8. J. Marshall Osborn, Commutative algebras satisfying an identity of degree four, Proc. Amer. Math. Soc. 16 (1965), 1114-1120.

UNIVERSITY OF IOWA 